\title{
Letters
}

\section{gMS-Classifier1 does not predict disability progression in multiple sclerosis}

Date received: 24 July 2018;

accepted: 9 August 2018

Several clinical, immunological and radiological biomarkers have been shown to predict the disease course of multiple sclerosis (MS). ${ }^{1-5}$ One potential serum marker is the gMS-Classifier1, which is composed of IgM anti-Glc antibodies, namely antiGAGA 2,3,4 and 6. Previous work demonstrated that the gMS-Classifier1 could not predict early conversion to clinically definite MS in a cohort of clinically isolated syndrome (CIS) patients, but predicted Expanded Disability Status Scale (EDSS) progression. Significance, however, was dependent on covariates, and confirmation in an independent study was required. ${ }^{3}$

The aim of this study was to test if the gMS-Classifier 1 could predict early disability progression in a large multicenter cohort of patients with CIS or relapse-onset MS.

Blood samples and clinical data were prospectively collected in four MS centers between 1993 and 2007: The Ottawa Hospital, Ottawa, Canada; Amsterdam University Medical Centers, the Netherlands; UMass Memorial Medical Center, Worcester, USA; and Hospital Ramón y Cajal, Madrid, Spain. Patients had a diagnosis of CIS $(n=118)$ or relapsing remitting multiple sclerosis (RRMS; $n=240$ ) at study onset. Age at blood sampling was between 18 and 50 years. Serum samples were stored frozen $<-70^{\circ} \mathrm{C}$ until assayed.

Baseline (EDSS) was performed within 6 months of blood sampling and was repeated during patient routine visits. Disability progression was defined as a sustained ( $\geqslant 6$ months) progression of at least 1.0 EDSS point over the baseline EDSS and progression to an EDSS score of 3.0 or higher.

Mean follow-up was 94 months.

In 2012, frozen serum samples were shipped to Glycominds Inc Lab (Simi Valley, CA, USA) for testing for anti-glycan antibodies as described before. ${ }^{3}$ If one of the antibodies was above the predefined cut-off (anti-GAGA2 >148.8 EIA units, anti-GAGA3 $>164.6$ EIA units, anti-GAGA4 $>133.6$ EIA units, and anti-GAGA6 $>168.1$ EIA units), patients were considered positive for the gMS-Classifier1. There were no significant differences for the key variables gMS-Classifier1 and EDSS progression between the four centers.

Of the 358 patients, 44 (12.3\%) were gMS-Classifier1 status positive. EDSS progression was available for 355 patients, of whom $158(44.5 \%)$ had confirmed progression at the end of follow-up. The percentage of patients showing EDSS progression did not differ between the groups, using the 1 point EDSS progression definition $(p=0.587)$ or for EDSS progression above $3.0 \quad(p=0.771)$. There was no association between EDSS progression and the gMS-Classifier1 $(p=0.778)$ or positive titres for any of the separate antibodies (anti-GAGA2: $p=0.934$, anti-GAGA3: $p=0.663$, anti-GAGA4: $p=0.712$, and anti-GAGA6: $p=0.440)$.

No statistical differences between the gMS-Classifier1 positive and negative group were observed for age $(p=0.631)$, disease duration $(p=0.147)$, gender $(p=0.154)$, baseline EDSS $(p=1.000)$, number of CIS patients at blood sampling ( $p=0.865)$, follow-up time $(p=0.587)$, relapse at blood sampling $(p=0.771)$, and steroids at blood sampling $(p=1.000)$.

Here, we present the results of a large cohort of patients from different centers from two continents, showing no statistical differences between gMS-Classifier1 positive and negative patients, convincingly indicating that the gMS-Classifier1 does not predict disability progression in MS.

\section{Declaration of Conflicting Interests}

The author(s) declared the following potential conflicts of interest with respect to the research, authorship, and/or publication of this article: Johannis A. van Rossum reports no conflicts of interest. Luisa M. Villar received payment for lecturing, travel expenses, research grants or consultancy from Merck-Serono, Biogen, Sanofi-Genzyme, Roche, and Novartis. Peter $\mathrm{N}$. Riskind is a site principal investigator for clinical trials with Hoffman-Laroche/Genentech, Biogen, and the National Multiple Sclerosis Society. Mark S. Freedman received educational grants from Genzyme
Multiple Sclerosis Journal 2019, Vol. 25(7) 1010-1011 DOI: 10.1177 1352458518798048 (C) The Author(s), 2018. (c) (i) (5) Article reuse guidelines: sagepub.com/journalspermissions 
Canada, honoraria or consultation fees from Actelion, BayerHealthcare, BiogenIdec, Chugai, Clene Nanomedicine, EMD Canada, Genzyme, Merck Serono, Novartis, Hoffman La-Roche, SanofiAventis, Teva Canada Innovation, is a member of a company advisory board, board of directors or other similar group for Actelion, BayerHealthcare, BiogenIdec, Clene Nanomedicine, Hoffman La-Roche, Merck Serono, MedDay, Novartis, SanofiAventis and participates in a company sponsored speaker's bureau of Sanofi-Genzyme. Charlotte Teunissen served on the advisory board of Fujirebio and Roche, received research consumables from Euroimmun, IBL, Fujirebio, Invitrogen, and Meso Scale Discovery, and performed contract research for IBL, Shire, Boehringer, Roche, and Probiodrug. Joep Killestein has accepted speaker and consultancy fees from Merck, Biogen, Teva, Genzyme, Roche and Novartis.

\section{Funding}

The author(s) declared receipt of the following financial support for the research, authorship, and/or publication of this article: Biomarker analysis for measurement of anti-glycan antibodies was funded and performed by Glycominds Incorporation.

\section{ORCID iD}

Johannis A van Rossum (D) https://orcid.org/00000002-0096-012X

\section{References}

1. Teunissen CE, Malekzadeh A, Leurs C, et al. Body fluid biomarkers for multiple sclerosis-The long road to clinical application. Nat Rev Neurol 2015; 11(10): 585-596.

2. Housley WJ, Pitt D and Hafler DA. Biomarkers in multiple sclerosis. Clin Immunol 2015; 161(1): 51-58.

\section{Analysis of Canadian multiple sclerosis patients does not support a role for FKBP6 in disease}

Date received: 21 June 2018; accepted: 30 August 2018

We read with interest a recent article by Mescheriakova et al. ${ }^{1}$ entitled Linkage analysis and whole exome sequencing identify a novel candidate gene in a Dutch multiple sclerosis family. In this study, the
3. Freedman MS, Metzig C, Kappos L, et al. Predictive nature of IgM anti-alpha-glucose serum biomarker for relapse activity and EDSS progression in CIS patients: A BENEFIT study analysis. Mult Scler 2012; 18(7): 966-973.

4. Martinelli V, Dalla Costa G, Messina MJ, et al. Multiple biomarkers improve the prediction of multiple sclerosis in clinically isolated syndromes. Acta Neurol Scand 2017; 136: 454-461.

5. Freedman MS, Laks J, Dotan N, et al. Anti-alphaglucose-based glycan IgM antibodies predict relapse activity in multiple sclerosis after the first neurological event. Mult Scler 2009; 15(4): 422-430.

\section{Johannis A van Rossum ${ }^{1}$ (D), Joep Killestein ${ }^{1}$, Luisa M Villar ${ }^{2}$, Peter N Riskind ${ }^{3}$, Mark S Freedman 4 , and Charlotte Teunissen ${ }^{5}$}

${ }^{1}$ Department of Neurology, Amsterdam Neuroscience, MS Center Amsterdam, VU University Medical Center Amsterdam, Amsterdam, The Netherlands ${ }^{2}$ Department of Neurology, Hospital Universitario Ramón y Cajal, Madrid, Spain

${ }^{3}$ Memorial Multiple Sclerosis Center, Department of Neurology, UMass Memorial Medical Center,

Worcester, MA, USA

${ }^{4}$ Division of Neurology, Department of Medicine, University of Ottawa, The Ottawa Hospital Research Institute, Ottawa, ON, Canada

${ }^{5}$ Department of Clinical Chemistry, Amsterdam

University Medical Centers, The Netherlands

\section{Correspondence to:}

\section{JA van Rossum}

Department of Neurology, Amsterdam Neuroscience, MS Center Amsterdam, VU University Medical Center Amsterdam, PO Box 7057, 1007 MB

Amsterdam, The Netherlands.

j.vanrossum@vumc.nl

authors described a missense variant in FKBP6 (p.R183C, rs147213094) co-segregating with multiple sclerosis (MS) in eight individuals from a large Dutch multi-incident family. Four healthy family members were also found to harbour this mutation, and it was not observed in one family member diagnosed with MS. Reduced penetrance and the presence of phenocopies does not detract from the authors' claim as familial forms of complex diseases, including MS, frequently are genetically heterogeneous. ${ }^{2-5}$ In addition, albeit not statistically significant,
Visit SAGE journals online journals.sagepub.com/ home/msj

(SAGE journals
Multiple Sclerosis Journal 2019, Vol. 25(7) 1011-1013 DOI: 10.1177 1352458518803789

(C) The Author(s), 2018. Article reuse guidelines: sagepub.com/journalspermissions 